Pacific

Journal of

Mathematics

SEVERAL SPLITTING CRITERIA FOR VECTOR BUNDLES AND REFLEXIVE SHEAVES

StePhen S.-T. Yau AND FeI Ye 


\title{
SEVERAL SPLITTING CRITERIA FOR VECTOR BUNDLES AND REFLEXIVE SHEAVES
}

\author{
StePhen S.-T. YAu And Fei Ye
}

\begin{abstract}
In this paper, we show two splitting criteria for vector bundles on complex projective spaces by analytic method. We also prove a splitting criterion for reflexive sheaves on Horrocks schemes by algebraic method.
\end{abstract}

\section{Introduction}

Vector bundles are favored objects studied in algebraic geometry and commutative algebra. We say that a vector bundle splits if it is isomorphic to a direct sum of line bundles. A very interesting problem is whether there are nonsplitting vector bundles of small ranks on complex projective spaces. Although such vector bundles exist in lower dimensions, they seem to be extremely rare as the dimension increases. In fact, Hartshorne conjectured:

Conjecture 1.1 [Hartshorne 1974]. If $n \geq 7$, all rank-2 vector bundles on the projective space $\mathbb{P}^{n}$ split.

Under some additional conditions, the conjecture was proved. However, the conjecture is still open. The most well-known condition is the vanishing of certain intermediate (local) cohomology groups. The first splitting criterion of this type is attributed to Horrocks.

Theorem 1.2 [Horrocks 1964]. Let $E$ be a vector bundle on the projective space $\mathbb{P}^{n}$ with $n \geq 2$. Then $E$ splits if and only if $H^{i}\left(\mathbb{P}^{n}, E(k)\right)=0$ for all $k \in \mathbb{Z}$ and $1 \leq i \leq n-1$.

A standard proof is to apply induction to $n$ and use the following so-called "restriction criterion".

Theorem 1.3. Let $E$ be a rank-r vector bundle over $\mathbb{P}^{n}$, with $n \geq 3$. E splits if and only if its restriction $\left.E\right|_{H}$ to some hyperplane $H \cong \mathbb{P}^{n-1} \subset \mathbb{P}^{n}$ splits.

The first author gratefully acknowledges the support from the National Nature Science Foundation of China (Grant No. 31271408) and Tsinghua University.

MSC2010: 14F05, 14J60, 32L10.

Keywords: holomorphic vector bundles, connection, reflexive sheaves, Horrocks scheme, splitting Criterion. 
Evans and Griffith improved Horrocks' criterion in the 1980's.

Theorem 1.4 [Evans and Griffith 1981]. Let E be a vector bundle on the projective space $\mathbb{P}^{n}$ of rank $r<n$. Then $E$ splits if and only if $H^{i}\left(\mathbb{P}^{n}, E(k)\right)=0$ for all $k \in \mathbb{Z}$ and $1 \leq i \leq r-1$.

Kumar, Peterson and Rao obtained another improvement of Horrocks' theorem. Theorem 1.5 [Kumar et al. 2003]. Let $E$ be a vector bundle on $\mathbb{P}^{n}$. If $\operatorname{rank} E<$ $2[n / 2]$, then $E$ splits if and only if $H^{i}\left(\mathbb{P}^{n}, E(k)\right)=0$ for all $k \in \mathbb{Z}$ and $1<i<n-1$.

Another type of splitting criteria involves extensibility of vector bundles. Let $X$ be an algebraic variety and $Y$ be a subvariety of $X$. A vector bundle $E$ on $Y$ is said to extend to $X$ if there exists a vector bundle $F$ on $X$ such that $\left.F\right|_{Y}=E$. Barth and van de Ven [1974] showed that a rank-2 vector bundle $E$ on $\mathbb{P}^{n}$ splits if and only if $E$ extends to $\mathbb{P}^{N}$ for all $N>n$. Their result was generalized to vector bundles of arbitrary rank by Sato [1977].

For any coherent sheaf $\mathscr{F}$, we denote the dual by $\mathscr{F}^{*}=\mathscr{H}$ om $\left(\mathscr{F}, \mathscr{O}_{X}\right)$. The next theorem combines extensibility and vanishing of cohomology groups.

Theorem 1.6 [Kempf 1990]. Let $E$ be a vector bundle on the projective space $\mathbb{P}^{n}$ with $n \geq 2$ and $E^{*}$ be its dual. Then $E$ splits if and only if the following two conditions are satisfied:

(1) E extends to $\mathbb{P}^{n+1}$.

(2) $H^{1}\left(\mathbb{P}^{n}, E \otimes E^{*}(-k)\right)=0$ for all positive integer $k$.

Proofs of the above mentioned theorems are all algebraic. However, the following remarkable criterion uses an analytic method.

Theorem 1.7 [Luk and Yau 1993]. Let $E$ be a holomorphic vector bundle on $\mathbb{C P}^{n}$ with $n \geq 2$ and $E^{*}$ be its dual. Then $E$ splits if and only if $H^{1}\left(\mathbb{C} \mathbb{P}^{n}, E \otimes E^{*}(k)\right)$ vanish for all $k \in \mathbb{Z}$.

In this paper, we employ Luk and Yau's idea to provide analytic proofs of some splitting criteria of vector bundles on complex projective spaces.

Let $p: \mathbb{C P}^{n+1} \backslash\{\xi\} \rightarrow \mathbb{C P}^{n}$ be the projection from a point $\xi \in \mathbb{C} \mathbb{P}^{n+1} \backslash \mathbb{C} \mathbb{P}^{n}$. We prove the following theorems in this paper.

Theorem A. A holomorphic vector bundle $E$ on $\mathbb{C P}^{n}$ splits if and only if $p^{*} E$ extends to a vector bundle on $\mathbb{C P}^{n+1}$.

Theorem B. Let $E$ be a holomorphic vector bundle on $\mathbb{C P}^{n}$. If $\operatorname{rank} E<2[n / 2]$, then $E$ splits if and only if the local cohomology groups $H_{\{\xi\}}^{i}\left(\mathbb{P}^{n+1}, \tilde{E}(k)\right)=0$ for all $k \in \mathbb{Z}$ and $1<i<n$, where $\tilde{E}$ is the extension of $p^{*} E$ on $\mathbb{C P}^{n+1}$.

Kumar observed that condition (2) in Theorem 1.6 implies that $p^{*} E$ extends to a vector bundle on $\mathbb{C} \mathbb{P}^{n+1}$. Thus Theorem A implies the following theorem. 
Theorem 1.8 [Kumar 2003]. Let $E$ be a holomorphic vector bundle on $\mathbb{C P}^{n}$ with $n \geq 2$ and $E^{*}$ be its dual. Then $E$ splits if and only if $H^{1}\left(\mathbb{C P}^{n}, E \otimes E^{*}(-k)\right)=0$ for all positive integer $k$.

On the other hand, generalizations of splitting criteria to reflexive sheaves and more general varieties have been obtained. Abe and Yoshinaga [2008] generalized the restriction criterion for reflexive sheaves on projective spaces. On the other hand, Bakhtary [2011] generalized the restriction criterion to Horrocks varieties.

Definition 1.9. A coherent sheaf $\mathscr{F}_{F}$ on $X$ is reflexive if $\mathscr{F}_{F} \cong \mathscr{F}^{* *}$. It is normal if for every open set $U \subset X$ and every closed subset $Y \subset U$ of codimension $\geq 2$, the restriction map $\mathscr{F}(U) \rightarrow \mathscr{F}(U \backslash Y)$ is bijective. We define the singular locus of $\mathscr{F}$ as $\operatorname{Sing}(\mathscr{F}):=\left\{x \in X \mid \mathscr{F}_{x}\right.$ is not locally free $\}$.

Definition 1.10. An algebraic variety $X$ is called a splitting variety if $H^{1}(X, L)=0$ for any line bundle $L$ on $X$. A Horrocks variety is a splitting variety $X$ with $H^{2}(X, L)=0$ for any line bundle $L$ on $X$.

In this paper, we prove a generalization of both the theorem of Bakhtary and the theorem of Abe and Yoshinaga.

Theorem C. Let $H$ be an effective ample divisor on a smooth projective variety $X$ of dimension $\operatorname{dim} X \geq 4$. Assume that $X$ is a Horrocks variety. Then a reflexive sheaf $\mathscr{F}$ on $X$ is splitting if and only if the restriction $\mathscr{F}_{H}{ }_{H}$ is splitting.

Theorems A and B will be proved in Section 2 and Theorem C will be proved in the last section.

\section{Splitting criteria via connections}

Let $E$ be a rank- $r$ complex vector bundle over a complex $n$-dimensional manifold $M$. Denote by $\mathscr{A}^{q}$ and $\mathscr{A}^{q}(E)$ the sheaves of smooth $q$-forms on $M$ and smooth $q$-forms on $M$ with coefficients in $E$ respectively.

Definition 2.1. A connection on $E$ is a $\mathbb{C}$-linear morphism

$$
\mathrm{D}: \mathscr{A}^{0}(E) \rightarrow \mathscr{A}^{1}(E)
$$

such that for any open subset $U$ of $M$

$$
\mathrm{D}(f \gamma)=\mathrm{d} f \otimes \gamma+f \mathrm{D}(\gamma),
$$

for any $f \in \Gamma\left(U, \mathscr{A}^{0}\right)$ and $\gamma \in \Gamma\left(U, \mathscr{A}^{0}(E)\right)$.

A connection $D$ of a vector bundle $E$ localized over any open subset $U$ is determined by a matrix $\omega=\left(\omega_{j}^{k}\right)$ of smooth 1-forms, called a connection matrix of $D$ over $U$. It is well known that if the complex vector bundle $E$ is holomorphic, then we have a connection $\mathrm{D}$ which can be decomposed into $\mathrm{D}^{\prime}+\bar{\partial}$ such that 
$\mathrm{D}^{\prime}: \mathscr{A}^{(0,0)}(E) \rightarrow \mathscr{A}^{(1,0)}(E)$ and $\bar{\partial}: \mathscr{A}^{(0,0)}(E) \rightarrow \mathscr{A}^{(0,1)}(E)$, where $\mathscr{A}^{(p, q)}(E)$ are the sheaves of smooth $(p, q)$-forms with coefficients in $E$.

Definition 2.2. Let $E$ be a holomorphic vector bundle over $\mathbb{C}^{n+1} \backslash\{0\}$ with a connection $\mathrm{D}=\mathrm{D}^{\prime}+\bar{\partial}$. Denote by $\left(\sum_{j=0}^{n} \theta_{i j}^{k} \mathrm{~d} z_{j}\right)$ the connection matrix of $\mathrm{D}^{\prime}$. We say $\mathrm{D}$ is holomorphic in the direction $z_{l}$ for some $0 \leq l \leq n$ if the $\theta_{i l}^{k}$ are holomorphic for all $1 \leq i, k \leq r$.

Theorem 2.8 of [Luk and Yau 1993] says that the existence of such a connection will force the cohomology groups $H^{i}\left(\mathbb{C}^{n+1} \backslash\{0\}, E\right)$ to vanish for $0<i<n$. To prove our theorems, we will look for such a connection.

Let $L$ be the line in $\mathbb{C}^{n+2}$ defined by $z_{0}=z_{1}=\cdots=z_{n}=0$ and $\xi=[0, \ldots, 0,1]$ be a point in $\mathbb{C} \mathbb{P}^{n+1} \backslash \mathbb{C P} \mathbb{P}^{n}$. Set $X=\mathbb{C}^{n+2} \backslash L$ and $U=\mathbb{C P}^{n+1} \backslash\{\xi\}$. Consider the projections $\pi: X \rightarrow U, \pi\left(\left(z_{0}, \ldots, z_{n+1}\right)\right)=\left[z_{0}, \ldots, z_{n+1}\right]$ and $p: U \rightarrow \mathbb{C P}^{n}$, $p\left(\left[z_{0}, \ldots, z_{n+1}\right]\right)=\left[z_{0}, \ldots, z_{n}\right]$. It is well known that $p$ defines a line bundle structure on $U$. In fact, $U$ is the total space of the line bundle $O_{\mathbb{C P}^{n}}(1)$.

Assume that $E$ is a holomorphic vector bundle on $\mathbb{C} \mathbb{P}^{n}$. We claim that $\pi^{*} p^{*} E$ has a connection which is holomorphic in the direction $z_{n+1}$. Let $D=D^{\prime}+\bar{\partial}$ be a connection on $E$. Assume that $D^{\prime}$ has a connection matrix $\omega^{i}=\left(\omega_{\beta}^{\alpha}\right)$ over $U_{i}=\left\{z_{i} \neq 0\right\} \subset \mathbb{C P}^{n}$, and $\omega_{\beta}^{\alpha}=\sum_{j=0}^{n} \theta_{\beta j}^{\alpha} \mathrm{d}\left(z_{j} / z_{i}\right)$. Pulling back $E$ and the connection $D$ to $X$, we see that $\pi^{*} p^{*} E$ admits a connection whose $D^{\prime}$-part is defined by the connection matrix

$$
\left(\sum_{j=0}^{n}\left(\theta_{\beta j}^{\alpha} \circ p \circ \pi\right) \frac{1}{z_{i}} \mathrm{~d} z_{j}-\sum_{j=0}^{n}\left(\theta_{\beta j}^{\alpha} \circ p \circ \pi\right) \frac{z_{j}}{\left(z_{i}\right)^{2}} \mathrm{~d} z_{i}\right),
$$

which is clearly holomorphic in the direction $z_{n+1}$.

Now we are ready to prove Theorems A and B. The next result is a special case of Lemma 2.2 in [Luk and Yau 1993].

\section{Lemma 2.3.}

$$
H^{i}\left(X, \pi^{*} p^{*} E\right)=\bigoplus_{k=-\infty}^{+\infty} H^{i}\left(U, p^{*} E(k)\right)
$$

Proof. Notice that the projection $\pi: X \rightarrow U$ admits a bundle structure whose fibers are the punctured complex line $\mathbb{C}^{*}$. In fact, one can check that $X$ is the total space of $O_{U}(1)$ with the zero section $U$ removed. Applying Lemma 2.2 of [Luk and Yau 1993], we get the equality.

Denote by $\iota: \mathbb{C} \mathbb{P}^{n+1} \backslash\{\xi\} \hookrightarrow \mathbb{C} \mathbb{P}^{n+1}$ the inclusion and $\tilde{E}=\iota_{*} p^{*} E$ the extension of $E$. The following proposition is the key to prove Theorems A and B.

Proposition 2.4. Assume that the local cohomology groups

$$
H_{\{\xi\}}^{i}\left(\mathbb{C} \mathbb{P}^{n+1}, \tilde{E}(k)\right)=H_{\{\xi\}}^{i+1}\left(\mathbb{C P}^{n+1}, \tilde{E}(k)\right)=0,
$$

for $0<i<n$ and all $k \in \mathbb{Z}$. Then $H^{i}\left(X, \pi^{*} p^{*} E\right)=0$ for $0<i<n$. 
Proof. By the assumption and the exact sequence of local cohomology

$$
\begin{aligned}
H_{\{\xi\}}^{i}\left(\mathbb{C} \mathbb{P}^{n+1}, \tilde{E}(k)\right) \rightarrow H^{i}\left(\mathbb{C} \mathbb{P}^{n+1}, \tilde{E}(k)\right) & \\
& \rightarrow H^{i}\left(U, p^{*} E(k)\right) \rightarrow H_{\{\xi\}}^{i+1}\left(\mathbb{C} \mathbb{P}^{n+1}, \tilde{E}(k)\right),
\end{aligned}
$$

we see that

$$
H^{i}\left(U, p^{*} E(k)\right)=H^{i}\left(\mathbb{C P}^{n+1}, \tilde{E}(k)\right) .
$$

Hence $H^{i}\left(U, p^{*} E(k)\right)$ is of finite dimension. By Serre vanishing theorem, there exists an integer $N$ such that $H^{i}\left(\mathbb{C} \mathbb{P}^{n+1}, \tilde{E}(-k)\right)=0$ for all integers $i, k$ with $|k| \geq N$ and $1 \leq i \leq n$. Therefore there are only finitely many $H^{i}\left(U, p^{*} E(-k)\right) \neq 0$. Thus $H^{i}\left(X, \pi^{*} p^{*} E\right)=\bigoplus_{k=-N}^{N} H^{i}\left(U, p^{*} E(-k)\right)$ is of finite dimension by Lemma 2.3.

We know that $\pi^{*} p^{*} E$ admits a connection which is holomorphic in the direction $z_{n+1}$. Applying the same argument as in the proof of Theorem 2.8 in [Luk and Yau 1993], we conclude that $H^{i}\left(X, \pi^{*} p^{*} E\right)=0$.

Theorem A follows very easily from Proposition 2.4.

Theorem A. Let $E$ be a holomorphic vector bundle on $\mathbb{C P}^{n}$. Then $E$ splits if and only if $p^{*} E$ extends to a vector bundle on $\mathbb{C} \mathbb{P}^{n+1}$.

Proof. Assume that $E=\bigoplus_{a} \mathscr{O}_{\mathbb{C P}^{n}}(a)$. Then $p^{*} \mathscr{O}_{\mathbb{C} \mathbb{P}^{n}}(a)$ extends uniquely to $\mathrm{O}_{\mathbb{C P}^{n+1}}(a)$. Hence $\tilde{E}$ is a vector bundle, moreover $\tilde{E}$ splits. Conversely, assume that $\tilde{E}$ is a vector bundle, then $\operatorname{depth}_{x} \tilde{E}=\operatorname{depth} \tilde{E}_{x}=\operatorname{dim} \widehat{O}_{x}=n+1$. By [Hartshorne 1967, Proposition 1.4 and Theorem 3.8], we know that $H_{\{\xi\}}^{i}\left(\mathbb{C} \mathbb{P}^{n+1}, \tilde{E}(-k)\right)=$ 0 for all $k \in \mathbb{Z}$ and $i \leq n$. By Proposition 2.4 and Lemma 2.3, we see that $H^{i}\left(U, p^{*} E(-k)\right)=0$ for all $k \in \mathbb{Z}$ and $1 \leq i \leq n$. Hence $H^{i}\left(\mathbb{C} \mathbb{P}^{n+1}, \tilde{E}(-k)\right)=0$ for all $k \in \mathbb{Z}$ and $1 \leq i \leq n$. It follows from Theorem 1.2 that $\tilde{E}$ splits, and so does $E$.

Another consequence of Proposition 2.4 is the following local cohomology version of Theorem 1.5 of Kumar, Peterson and Rao.

Theorem B. Let $E$ be a vector bundle on $\mathbb{C P}^{n}$. If $\operatorname{rank} E<2[n / 2]$, then $E$ splits if and only if for all $k \in \mathbb{Z}$ and $1<i<n$ the local cohomology groups $H_{\{\xi\}}^{i}\left(\mathbb{C} \mathbb{P}^{n+1}, \tilde{E}(k)\right)=0$.

Proof. It is clear that if $E$ splits then $H_{\{\xi\}}^{i}\left(\mathbb{C} \mathbb{P}^{n+1}, \tilde{E}(k)\right)=0$ for all $k \in \mathbb{Z}$ and $1<i<n$. Assume that $H_{\{\xi\}}^{i}\left(\mathbb{C} \mathbb{P}^{n+1}, \tilde{E}(k)\right)=0$ for all $k \in \mathbb{Z}$ and $1<i<n$. By Proposition 2.4,

$$
H^{i}\left(X, \pi^{*} p^{*} E\right)=\bigoplus_{k=-\infty}^{+\infty} H^{i}\left(U, p^{*} E(-k)\right)=0
$$

for $2 \leq i \leq n-2$. In particular, $H^{i}\left(U, p^{*} E\right)=\bigoplus_{k=-\infty}^{+\infty} H^{i}\left(\mathbb{C P}^{n}, E(-k)\right)=0$ for $2 \leq i \leq n-2$. By Theorem 1.5, we see that $E$ splits. 
Another local version of Theorem 1.5 was obtained as one of the main theorems in [Majidi-Zolbanin 2005].

\section{Splitting of reflexive sheaves}

The proof of Theorem $\mathrm{C}$ relies on the following two propositions.

Proposition 3.1. Let $\mathscr{F}$ be a reflexive sheaf on a smooth projective variety $X$ and $H$ be an effective ample divisor $X$. Assume that $H$ is a splitting variety. If $\left.\mathscr{F}_{\mid}\right|_{H}$ splits into a direct sum of line bundles, then

$$
H^{1}(X, \mathscr{F}(k H))=0 \text { for all } k \in \mathbb{Z} \text {. }
$$

Proof. By assumption, we have a surjective morphism

$$
H^{1}(X, \mathscr{F}((k-1) H)) \rightarrow H^{1}(X, \mathscr{F}(k H)) \rightarrow 0 .
$$

By Serre duality, $H^{1}(X, \mathscr{F}(k H)) \cong \operatorname{Hom}\left(\operatorname{Ext}^{n-1}\left(\mathscr{F}(k H), \omega_{X}\right), k\right)$. It suffices to show that $\operatorname{Ext}^{n-1}\left(\mathscr{F}(k H), \omega_{X}\right)=0$ for $k \ll 0$. Consider the spectral sequence of local and global Ext functors

$$
E_{2}^{p, q}=H^{p}\left(X, \mathscr{E} \mathrm{Xt}^{q}\left(\mathscr{F}, \omega_{X}\right)\right) \Rightarrow E^{p+q}=\operatorname{Ext}^{p+q}\left(\mathscr{F}, \omega_{X}\right) .
$$

Since $\left.\mathscr{F}\right|_{H}$ is free, then $\operatorname{Sing}\left(\left.\mathscr{F}\right|_{H}\right)=\varnothing$, which implies that $\operatorname{Sing}(\mathscr{F}) \cap H=\varnothing$. Note that the singular locus $\operatorname{Sing}(\mathscr{F})$ is a closed subset of $X$ and $H$ is ample. If $\operatorname{dim} \operatorname{Sing}(\mathscr{F})=d>0$, then $\operatorname{Sing}(\mathscr{F}) \cdot H^{d}>0$. In particular, $\operatorname{Sing}(\mathscr{F}) \cap H \neq \varnothing$. Therefore, $\operatorname{dim} \operatorname{Sing}(\mathscr{F})=0$. Since a coherent sheaf $\mathscr{F}$ is free at a point $p \in X$ if and only if the stalk $\left(\mathscr{E} \mathrm{xt}^{q}\left(\mathscr{F}_{F}, \mathscr{G}\right)\right)_{p}=\operatorname{Ext}^{q}\left(\mathscr{F}_{p}, \mathscr{G}_{p}\right)=0$ for all $q>0$ and any coherent sheaf $\mathscr{G}$, we see that $\operatorname{dim} \operatorname{Supp}\left(\mathscr{E} \mathrm{xt}^{q}\left(\mathscr{F}, \omega_{X}\right)\right)=0$ for $q>0$. Hence, $H^{p}\left(X, \mathscr{E} \mathrm{xt}^{q}\left(\mathscr{F}_{F}, \omega_{X}\right)\right)=0$ for $p>0$ and $q>0$. Now there are only two $E^{2}$-terms $H^{0}\left(X, \mathscr{E} \mathrm{Xt}^{n-1}\left(\mathscr{F}, \omega_{X}\right)\right)$ and $H^{n-1}\left(X, \mathscr{H} \operatorname{om}\left(\mathscr{F}, \omega_{X}\right)\right)$ which may contribute to $\operatorname{Ext}^{n-1}\left(\mathscr{F}, \omega_{X}\right)$. Since $\mathscr{F}$ is reflexive, then $\operatorname{depth}_{x} \mathscr{F} \geq 2$ for all $x \in X$ by [Hartshorne 1980, Proposition 1.3]. Thus $H_{\{x\}}^{1}\left(\mathscr{F}_{x}\right)=0$. By local duality, we get $\mathscr{E}_{\mathrm{Xt}}^{n-1}\left(\mathscr{F}_{x}, \omega_{X, x}\right)=H_{\{x\}}^{1}\left(\mathscr{F}_{x}\right)=0$. Hence ${ }^{\mathscr{E}} \mathrm{xt}^{n-1}\left(\mathscr{F}_{F}, \omega_{X}\right)=0$. The spectral sequence then tells us that there is a surjective morphism

$$
H^{n-1}\left(X, \mathscr{H} \operatorname{om}\left(\mathscr{F}, \omega_{X}\right)\right) \rightarrow \operatorname{Ext}^{n-1}\left(\mathscr{F}, \omega_{X}\right) .
$$

Since $H^{n-1}\left(X, \mathscr{F}^{*}(-k H) \otimes \omega_{X}\right)=0$ for $-k \gg 0$ by Serre vanishing theorem, replacing $\mathscr{F}$ by $\mathscr{F}(k H)$ in $(* *)$, we conclude that

$$
\operatorname{Ext}^{n-1}\left(\mathscr{F}(k H), \omega_{X}\right)=0 \quad \text { for all } k \ll 0 .
$$

Theorem 3.2. Let $\mathscr{E}$ be a vector bundle and $\mathscr{F}$ be a reflexive sheaf over a smooth projective variety $X$ of dimension $\operatorname{dim} X \geq 4$. Let $H$ be an effective ample divisor on $X$. If $\left.\left.\mathscr{F}\right|_{H} \cong \mathscr{E}\right|_{H}$ and $H^{1}\left(X, \mathscr{E}^{*} \otimes \mathscr{F}(-H)\right)=0$, then $\mathscr{F} \cong \mathscr{E}$. 
Proof. By the assumption, we see that $\mathscr{H} \mathrm{om}(\mathscr{E}, \mathscr{F})=\mathscr{E}^{*} \otimes \mathscr{F}$ is also reflexive. Since a reflexive sheaf is torsion free, then the following sequence is exact:

$$
\left.0 \rightarrow \mathscr{E}^{*} \otimes \mathscr{F}(-H) \rightarrow \mathscr{E}^{*} \otimes \mathscr{F} \rightarrow\left(\mathscr{E}^{*} \otimes \mathscr{F}\right)\right|_{H} \rightarrow 0 .
$$

Since $H^{1}\left(X, \mathscr{E}^{*} \otimes \mathscr{F}(-H)\right)=0$, then $H^{0}\left(X, \mathscr{E}^{*} \otimes \mathscr{F}\right) \rightarrow H^{0}\left(H,\left.\left(\mathscr{E}^{*} \otimes \mathscr{F}\right)\right|_{H}\right)$ is surjective. Note that $\operatorname{Hom}(\mathscr{E}, \mathscr{F})=H^{0}(X, \mathscr{H}$ om $(\mathscr{E}, \mathscr{F}))$. Therefore, there is a morphism $\varphi: \mathscr{E} \rightarrow \mathscr{F}$ extending the isomorphism $\phi:\left.\left.\mathscr{E}\right|_{H} \rightarrow \mathscr{F}\right|_{H}$. We need to show that $\varphi$ is an isomorphism. Consider $\operatorname{det} \varphi: \operatorname{det} \mathscr{E} \rightarrow \operatorname{det} \mathscr{F}$. Since $\left.\left.\mathscr{E}\right|_{H} \cong \mathscr{F}\right|_{H}$ and $\operatorname{Pic}(X)=$ $\operatorname{Pic}(H)$ by Grothendieck-Lefschetz theorem, we conclude that det $\mathscr{E}=\mathscr{O}_{X}\left(c_{1}(\mathscr{E})\right)=$ $\mathcal{O}_{X}\left(c_{1}(\mathscr{F})\right)=\operatorname{det} \mathscr{F}$. Therefore $\operatorname{det} \varphi \in H^{0}\left(X, \operatorname{det} \mathscr{E}^{*} \otimes \operatorname{det} \mathscr{F}\right)=\mathbb{C}$ because a reflexive rank-1 sheaf is a line bundle (see [Okonek et al. 1980, Lemma 1.1.15]). Clearly, $\operatorname{det} \varphi$ is a nonzero constant, since $\operatorname{det} \phi$ is a nonzero constant. Thus at each $x \in X \backslash\left(\operatorname{Sing}\left(\mathscr{E}^{*} \otimes \mathscr{F}\right)\right)$, the morphism $\varphi_{x}$ is an isomorphism. Since $\mathscr{E}^{*} \otimes \mathscr{F}^{*}$ is reflexive, then $\operatorname{codim}\left(\operatorname{Sing}\left(\mathscr{C}^{*} \otimes \mathscr{F}\right)\right) \geq 3$ and hence $\varphi$ is an isomorphism by [Hartshorne 1980, Proposition 1.6].

Theorem C follows easily from Proposition 3.1 and Theorem 3.2.

Theorem C. Let $H$ be an effective ample divisor on a smooth projective variety $X$ of dimension $\operatorname{dim} X \geq 4$. Assume that $X$ is a Horrocks variety. Then a reflexive sheaf $\mathscr{F}$ on $X$ is splitting if and only if the restriction $\left.\mathscr{F}_{\mid}\right|_{H}$ is splitting.

Proof. By [Bakhtary 2011, Proposition 4.13], $X$ is Horrocks if and only if $X$ and all effective ample divisors are splitting. Clearly, if $\mathscr{F}$ is splitting, then $\left.\mathscr{F}_{H}\right|_{H}$ is splitting. Conversely, assume that $\left.\mathscr{F}\right|_{H}$ is splitting. By Grothendieck-Lefschetz theorem, there is a splitting vector bundle $\mathscr{E}$ on $X$ such that $\left.\mathscr{E}\right|_{H}=\left.\mathscr{F}\right|_{H}$. By Proposition 3.1, we know that $H^{1}\left(X, \mathscr{E}^{*} \otimes \mathscr{F}(-H)\right)=0$. Therefore, $\mathscr{E} \cong \mathscr{F}$ by Theorem 3.2.

In [Abe and Yoshinaga 2008], the authors also generalized Horrocks cohomology criterion for reflexive sheaves on projective spaces. However, Horrocks cohomology criterion may not hold on Horrocks varieties in general. There exist smooth hypersurfaces in $\mathbb{P}^{5}$ with nonsplit vector bundles satisfying the Horrocks cohomology condition (see Remarks in the introduction of [Kumar et al. 2007]). It will be very interesting to know under what cohomology conditions a vector bundle on a Horrocks variety splits.

\section{References}

[Abe and Yoshinaga 2008] T. Abe and M. Yoshinaga, "Splitting criterion for reflexive sheaves", Proc. Amer. Math. Soc. 136:6 (2008), 1887-1891. MR 2009f:14029 Zbl 1139.14034

[Bakhtary 2011] P. Bakhtary, "Splitting criteria for vector bundles on higher-dimensional varieties", Pacific J. Math. 252:1 (2011), 19-29. MR 2862139 Zbl 1228.14047

[Barth and Van de Ven 1974] W. Barth and A. Van de Ven, "A decomposability criterion for algebraic 2-bundles on projective spaces”, Invent. Math. 25 (1974), 91-106. MR 52 \#420 Zbl 0295.14006 
[Evans and Griffith 1981] E. G. Evans and P. Griffith, "The syzygy problem", Ann. of Math. (2) 114:2 (1981), 323-333. MR 83i:13006 Zbl 0497.13013

[Hartshorne 1967] R. Hartshorne, Local cohomology: a seminar given by A. Grothendieck, Harvard University, Fall, 1961, Lecture Notes in Mathematics 41, Springer, Berlin, 1967. MR 37 \#219 Zbl 0185.49202

[Hartshorne 1974] R. Hartshorne, "Varieties of small codimension in projective space", Bull. Amer. Math. Soc. 80 (1974), 1017-1032. MR 52 \#5688 Zbl 0304.14005

[Hartshorne 1980] R. Hartshorne, "Stable reflexive sheaves", Math. Ann. 254:2 (1980), 121-176. MR 82b:14011 Zbl 0431.14004

[Horrocks 1964] G. Horrocks, "Vector bundles on the punctured spectrum of a local ring", Proc. London Math. Soc. (3) 14 (1964), 689-713. MR 30 \#120 Zbl 0126.16801

[Kempf 1990] G. R. Kempf, "A criterion for the splitting of a vector bundle", Forum Math. 2:5 (1990), 477-480. MR 91j:14036 Zbl 0715.14004

[Kumar 2003] N. M. Kumar, "Vector bundles on projective spaces", pp. 185-188 in Advances in algebra and geometry (Hyderabad, 2001), edited by C. Musili et al., Hindustan Book Agency, New Delhi, 2003. MR 2004f:14059 Zbl 1031.14007

[Kumar et al. 2003] N. M. Kumar, C. Peterson, and A. P. Rao, "Monads on projective spaces", Manuscripta Math. 112:2 (2003), 183-189. MR 2005g:14083 Zbl 1030.14008

[Kumar et al. 2007] N. M. Kumar, A. P. Rao, and G. V. Ravindra, "Arithmetically Cohen-Macaulay bundles on hypersurfaces", Comment. Math. Helv. 82:4 (2007), 829-843. MR 2008f:14059 Zbl 1131.14047

[Luk and Yau 1993] H. S. Luk and S. S.-T. Yau, "Cohomology and splitting criterion for holomorphic vector bundles on CP" ", Math. Nachr. 161 (1993), 233-238. MR 94k:14037 Zbl 0809.14013

[Majidi-Zolbanin 2005] M. Majidi-Zolbanin, Splitting of vector bundles on punctured spectrum of regular local rings, Ph.D. thesis, City University of New York, 2005, Available at http:// search.proquest.com/docview/305005817. MR 2707777

[Okonek et al. 1980] C. Okonek, M. Schneider, and H. Spindler, Vector bundles on complex projective spaces, Progress in Mathematics 3, Birkhäuser, Boston, 1980. MR 81b:14001 Zbl 0438.32016

[Sato 1977] E.-i. Sato, "On the decomposability of infinitely extendable vector bundles on projective spaces and Grassmann varieties”, J. Math. Kyoto Univ. 17:1 (1977), 127-150. MR 55 \#10459 Zbl 0362.14005

Received October 13, 2012.

STEPHEN S.-T. YAU

DEPARTMENT OF MATHEMATICAL SCIENCES

TSINGHUA UNIVERSITY

Room A-203, ScIENCE BuILding

BEIJING, 100084

CHINA

yau@uic.edu

FEI Ye

DePartment of MATHEMATICS

The University of Hong Kong

POKFULAM

HONG Kong

fye@maths.hku.hk 


\title{
PACIFIC JOURNAL OF MATHEMATICS
}

\author{
msp.org/pjm
}

Founded in 1951 by E. F. Beckenbach (1906-1982) and F. Wolf (1904-1989)

\section{EDITORS}

V. S. Varadarajan (Managing Editor)

Department of Mathematics

University of California

Los Angeles, CA 90095-1555

pacific@math.ucla.edu

Paul Balmer

Department of Mathematics

University of California

Los Angeles, CA 90095-1555

balmer@math.ucla.edu

Daryl Cooper

Department of Mathematics

University of California

Santa Barbara, CA 93106-3080 cooper@math.ucsb.edu

Jiang-Hua Lu

Department of Mathematics

The University of Hong Kong

Pokfulam Rd., Hong Kong jhlu@maths.hku.hk
Don Blasius

Department of Mathematics University of California

Los Angeles, CA 90095-1555

blasius@math.ucla.edu

Robert Finn

Department of Mathematics Stanford University

Stanford, CA 94305-2125

finn@math.stanford.edu

Sorin Popa

Department of Mathematics

University of California

Los Angeles, CA 90095-1555 popa@math.ucla.edu

Paul Yang

Department of Mathematics Princeton University

Princeton NJ 08544-1000

yang@math.princeton.edu

\section{PRODUCTION}

Silvio Levy, Scientific Editor, production@msp.org

\section{SUPPORTING INSTITUTIONS}

ACADEMIA SINICA, TAIPEI

CALIFORNIA INST. OF TECHNOLOGY

INST. DE MATEMÁTICA PURA E APLICADA

KEIO UNIVERSITY

MATH. SCIENCES RESEARCH INSTITUTE

NEW MEXICO STATE UNIV.

OREGON STATE UNIV.

\author{
STANFORD UNIVERSITY \\ UNIV. OF BRITISH COLUMBIA \\ UNIV. OF CALIFORNIA, BERKELEY \\ UNIV. OF CALIFORNIA, DAVIS \\ UNIV. OF CALIFORNIA, LOS ANGELES \\ UNIV. OF CALIFORNIA, RIVERSIDE \\ UNIV. OF CALIFORNIA, SAN DIEGO \\ UNIV. OF CALIF., SANTA BARBARA
}

\author{
Vyjayanthi Chari \\ Department of Mathematics \\ University of California \\ Riverside, CA 92521-0135 \\ chari@math.ucr.edu \\ Kefeng Liu \\ Department of Mathematics \\ University of California \\ Los Angeles, CA 90095-1555 \\ liu@math.ucla.edu \\ Jie Qing \\ Department of Mathematics \\ University of California \\ Santa Cruz, CA 95064 \\ qing@cats.ucsc.edu
}

These supporting institutions contribute to the cost of publication of this Journal, but they are not owners or publishers and have no responsibility for its contents or policies.

See inside back cover or msp.org/pjm for submission instructions.

The subscription price for 2013 is US \$400/year for the electronic version, and \$485/year for print and electronic.

Subscriptions, requests for back issues and changes of subscribers address should be sent to Pacific Journal of Mathematics, P.O. Box 4163, Berkeley, CA 94704-0163, U.S.A. The Pacific Journal of Mathematics is indexed by Mathematical Reviews, Zentralblatt MATH, PASCAL CNRS Index, Referativnyi Zhurnal, Current Mathematical Publications and Web of Knowledge (Science Citation Index).

The Pacific Journal of Mathematics (ISSN 0030-8730) at the University of California, c/o Department of Mathematics, 798 Evans Hall \#3840, Berkeley, CA 94720-3840, is published twelve times a year. Periodical rate postage paid at Berkeley, CA 94704, and additional mailing offices. POSTMASTER: send address changes to Pacific Journal of Mathematics, P.O. Box 4163, Berkeley, CA 94704-0163.

PJM peer review and production are managed by EditFLOW ${ }^{\circledR}$ from Mathematical Sciences Publishers.

\section{PUBLISHED BY}

mathematical sciences publishers

nonprofit scientific publishing

http://msp.org/

(C) 2013 Mathematical Sciences Publishers 


\section{PACIFIC JOURNAL OF MATHEMATICS}

Volume $266 \quad$ No. $2 \quad$ December 2013

Rate of attraction for a semilinear wave equation with variable coefficients and

critical nonlinearities

FÁGNER DiAs ARARUNA and FLANK DAVID MORAIS BEZERRA

The Brin-Thompson groups $s V$ are of type $\mathrm{F}_{\infty}$

Martin G. Fluch, Marco Marschler, Stefan Witzel and

MATTHEW C. B. ZAREMSKY

Ideal decompositions of a ternary ring of operators with predual

MASAYOSHI KANEDA

A study of real hypersurfaces with Ricci operators in 2-dimensional complex space 305 forms

Dong Ho Lim, WoOn Ha SoHn and Hyunjung Song

On commensurability of fibrations on a hyperbolic 3-manifold

HideTOSHI MASAI

Multiplicative Dirac structures

CRISTIÁN ORTIZ

On the finite generation of a family of Ext modules

TONY J. PuthenPuRAKAL

Index formulae for Stark units and their solutions

XAVIER-FranÇOIS ROBLOT

The short time asymptotics of Nash entropy

GUOYI XU

Several splitting criteria for vector bundles and reflexive sheaves

STEPHEN S.-T. YAU and FEI YE

The minimal volume orientable hyperbolic 3-manifold with 4 cusps

KEN'ICHI YOSHIDA

On the Witten rigidity theorem for string ${ }^{c}$ manifolds

JIANQING YU and BO LIU 